\title{
Oral direct inhibitors of coagulation
}

\author{
HG Watson \\ Consultant Haematologist, Department of Haematology, Aberdeen Royal Infirmary, Aberdeen, UK
}

\begin{abstract}
For the first time in 50 years new oral anticoagulants of proven efficacy and with acceptable safety profiles are available for patients with atrial fibrillation and venous thromboembolism. Here is a brief overview of the benefits and possible disadvantages of using these drugs.
\end{abstract}

KEYWORDS Dabigatran, rivaroxaban, apixaban, anticoagulation, atrial fibrillation, venous thromboembolism

DECLARATIONS OF INTERESTS No conflicts of interest declared.

\author{
Correspondence to HG Watson \\ Department of Haematology \\ Aberdeen Royal Infirmary \\ Aberdeen, AB25 2ZN UK \\ e-mail
}

henrywatson@nhs.net

\section{OVERVIEW}

Until very recently coumarins were the only group of drugs that had been successfully used for long-term oral anticoagulation. In the UK warfarin is the main drug from this group that is used, while in other parts of the world acenocoumarol, phenindione, and phenprocoumon are preferred. All of these drugs are vitamin $\mathrm{K}$ antagonists that prevent the gamma carboxylation of the vitamin K-dependent coagulation factors (factors II, VII, IX, and $X)$, which renders these factors unable to participate in blood coagulation. However, because of huge variability in the dose effect of these drugs between and within patients, a method of monitoring their effect in individual patients had to be developed. The international normalised ratio (INR) is a standardised method of describing the level of anticoagulation achieved by a coumarin. The use of an international sensitivity index (ISI), which is assigned to the thromboplastins used to perform the prothrombin time, assures that INR tests performed in different laboratories, using different analysers, and different thromboplastins should give approximately the same value, irrespective of where a sample is tested. In spite of this development, anticoagulant control of coumarins is a major challenge. The dose of warfarin required is very dependent on polymorphisms in two enzyme systems involved with the mode of action of and the metabolism of coumarins (VKORC-I and CYP 2C9). In addition, dietary vitamin K intake has a significant effect on coumarin dosing and the coumarins have huge numbers of drug interactions with many classes of drugs. All of these features make the management of patients on coumarins complicated.'

For many years clinicians managing patients requiring anticoagulation have suggested that the optimal anticoagulant for long-term use should be one that is taken by the oral route in a fixed dose, has a rapid onset of action, a half-life that is compatible with once or twice daily dosing, has few drug interactions, and does not require monitoring of its anticoagulant effect. That set of requirements has been satisfied by new anticoagulants that directly inhibit the key active coagulation factors thrombin and factor $\mathrm{Xa}$. These drugs have emerged from an extensive process of development and, following evaluation in clinical trials, have been licensed for use in the prevention of peri-operative venous thromboembolism following high-risk orthopaedic surgery; for the prevention of stroke and other forms of systemic embolism in patients with high-risk atrial fibrillation; and for the treatment and long-term prevention of deep vein thrombosis and pulmonary embolism. The rest of this article looks at these new drugs in a bit more depth, reviewing their pharmacology and the results of the clinical studies that have led to their licensing. In addition I will review areas of controversy and potential issues that might complicate the use of these agents in comparison with warfarin, the drug that they are replacing in many instances. These will include the management of bleeding, especially in view of the present lack of specific reversal strategies for the new agents, the management of the perioperative period in patients taking these drugs, and the interpretation of routine coagulation tests that are variably prolonged by these drugs.

\section{KEY FEATURES OF DABIGATRAN ETEXILATE, APIXABAN, AND RIVAROXABAN}

These drugs differ from warfarin in many ways. Their mechanism of action is by the direct inhibition of a single activated coagulation factor, either thrombin (dabigatran etexilate) or activated factor $X$ (FXa: apixaban and rivaroxaban), rather than the indirect inhibition of the function of numerous coagulation factors seen with the coumarins. Unlike warfarin, which requires 3-7 days to become established at therapeutic levels, these drugs have a rapid onset of action with the patient being fully anticoagulated within 2-3 hours of the ingestion of any of these new 
agents. As such, in acute situations it is possible to administer the new agents without use of initial parenteral anticoagulant (usually unfractionated or low molecular weight heparin [LMWH]).

As indicated earlier there are many variables that affect the action of warfarin. This results in a situation where predicting the dose of warfarin is problematic and aided only in a limited way by methods such as the Fennerty dosing chart. One of the major advantages of the oral direct inhibitors (ODI) is that for the vast majority of patients a fixed dose produces a similar level of anticoagulation (measured as the drug concentration in the blood). This seems to hold true across age, sex, race, and size differences. There are issues around drug concentrations in patients with significant renal impairment and dabigatran is contraindicated in patients with a creatinine clearance of less than $30 \mathrm{ml} / \mathrm{min}$, while rivaroxaban and apixaban are contraindicated in patients with a glomerular filtration rate (GFR) less than $15 \mathrm{ml} /$ $\mathrm{min}$. Caution is advised in patients with lesser degrees of renal impairment. Likewise, these agents are contraindicated in severe liver impairment and relatively contraindicated in patients with significant hepatic impairment as indicated by liver enzymes $>2 x$ upper limit of normal and Child-Pugh scores B and C.

As a general feature, groups in which caution is required include those of a low body weight, the very elderly, and those with impaired renal and hepatic function. Obviously patients who are taking other medications that influence haemostasis such as anti-platelet and non-steroidal anti-inflammatory drugs require some consideration before administering any anticoagulant. Few drugs have significant interactions with these new agents. Drugs which inhibit both P-glycoprotein and CYP 3A4, including protease inhibitors and azoles, result in significant increases in apixaban and rivaroxaban plasma levels while drugs that induce the action of both, like rifampicin, carbamazepine, phenytoin, phenobarbital and St John's Wort result in significant reductions in plasma levels. Strong P-glycoprotein inhibitors such as azoles, cyclosporine, and tacrolimus are contraindicated in patients receiving dabigatran etexilate while moderate inhibitors such as verapamil, quinidine, amiodarone, and ticagrelor are relatively contraindicated. ${ }^{2}$

\section{OUTSTANDING ISSUES FOR THE NEW ORAL DIRECT INHIBITORS}

Along with the benefits that are discussed above the new ODls have some drawbacks that have direct relevance to clinical practice. The major issues relate to the lack of a specific antidote to use in the event of major or life-threatening bleeding, the lack of awareness of the poor correlation between the concentrations of these drugs in plasma and routinely used coagulation tests, such as the prothrombin time (PT) and the activated partial thromboplastin time (APTT), and the need to develop new protocols for the discontinuation of these anticoagulants around periods of minor or major surgical intervention.

Warfarin has a specific antidote, vitamin K, that along with appropriate coagulation factor replacement (prothrombin complex concentrate) reliably reverses the coagulopathy. The ODls have no such targeted reversal strategy at present although they have relatively short half-lives which is of some advantage in the case of bleeding (around 9-14 hours). Treatment of major bleeding is supportive and includes endoscopic and surgical methods of controlling bleeding combined with the use of pro-haemostatic agents such as tranexamic acid and prothrombin complex concentrate. There are, however, interesting developments on the horizon and it is likely that specific strategies for the reversal of these agents will become available in the next few years. Of note, in general, the incidence of major bleeding was similar and there was no difference in the rates of fatal haemorrhage in the clinical trials where apixaban, rivaroxaban, and dabigatran etexilate were compared with warfarin for the management of venous thromboembolism and atrial fibrillation.

Over the past ten years there has been quite a lot of work looking at the most appropriate ways of managing the peri-surgical period in patients on warfarin. In general this consists of an assessment of the relative risks of thrombosis associated with temporary discontinuation of warfarin, combined with an assessment of the bleeding risk of continuation or early re-introduction of warfarin. For surgery with a high bleeding risk warfarin is discontinued five days prior to the procedure and $\mathrm{LMWH}$ is introduced soon after surgery with a decision being made about the use of heparins prior to the surgery. The need for early re-introduction of escalating doses of heparin or $\mathrm{LMWH}$ is made depending on the perceived thrombosis risk for the patient, which consists of the indication for anticoagulation and the thrombosis risk associated with the surgical procedure. For low bleeding risk surgery, such as most routine dental procedures and cataract surgery, there is no requirement to discontinue warfarin if the INR is satisfactory. Similar considerations are now being made for patients on the ODls. Where discontinuation is required, the timing of this prior to surgery can be decided by calculating the elimination half-life of the drug being used. This is best performed by considering the pharmacokinetics of the drug and the patient's renal function.

The final issue to be aware of is the lack of correlation between the state of anticoagulation of a patient and the results of routine coagulation tests. Unlike warfarin, 
TABLE I Advantages and issues of oral direct inhibitors

\begin{tabular}{|c|c|}
\hline Key advantages for ODI & Potential issues for ODI \\
\hline $\begin{array}{l}\text { Fixed oral dosing, for most } \\
\text { groups of patients. } \\
\text { Renal function is a key issue } \\
\text { for dosing. There are few } \\
\text { data around use of these } \\
\text { agents in very high body } \\
\text { weight patients. }\end{array}$ & $\begin{array}{l}\text { Lack of specific antidote. } \\
\text { Presently, bleeding is } \\
\text { managed by supportive } \\
\text { strategies. Active work is in } \\
\text { progress to develop specific } \\
\text { strategies. }\end{array}$ \\
\hline $\begin{array}{l}\text { Rapid onset of action and } \\
\text { pharmacokinetics suitable } \\
\text { for daily or twice daily } \\
\text { dosing. Peak plasma levels } \\
2-4 \text { hours post dosing. }\end{array}$ & $\begin{array}{l}\text { Variable effects on routine } \\
\text { coagulation tests. } \\
\text { Thrombin time and APTT } \\
\text { tests are sensitive to the } \\
\text { effects of dabigatran- } \\
\text { accurate measurement } \\
\text { should be by haemoclot } \\
\text { assay. } \\
\text { PT tests are moderately } \\
\text { sensitive to rivaroxaban- } \\
\text { accurate measurement } \\
\text { should be by an } \\
\text { appropriately calibrated anti- } \\
\text { Xa assay. } \\
\text { Apixaban has little effect on } \\
\text { APTT and PT. } \\
\text { Accurate assessment should } \\
\text { be by an appropriately } \\
\text { calibrated anti-Xa assay. }\end{array}$ \\
\hline Few drug interactions. & $\begin{array}{l}\text { Lack of experience } \\
\text { especially in peri-surgical } \\
\text { situation. }\end{array}$ \\
\hline $\begin{array}{l}\text { No need for routine } \\
\text { monitoring of coagulation. } \\
\text { Indications for measuring } \\
\text { drug levels accurately might } \\
\text { include emergency surgery, } \\
\text { development of renal failure } \\
\text { and active bleeding. }\end{array}$ & $\begin{array}{l}\text { Drug costs high but slightly } \\
\text { offset by the removal of } \\
\text { monitoring of anticoagulant } \\
\text { effect. }\end{array}$ \\
\hline
\end{tabular}

which predictably prolongs the prothrombin time, apixaban, rivaroxaban, and dabigatran have varied effects on the PT and the APTT.This is potentially a major issue and doctors responsible for patients admitted on these drugs should seek advice from the haematology laboratory and clinical colleagues in situations where an invasive procedure is planned. ${ }^{3}$

\section{SUMMARY}

The considerations with regard to oral direct inhibitors are summarised in Table I.

\section{Highlights}

- Oral direct inhibitors (ODIs) are taken in fixed doses.

- Routine monitoring of anticoagulant effect is not required for patients on the ODIs.

- Efficacy is similar to that seen with warfarin in patients with atrial fibrillation and venous thromboembolism.

- There are no specific drug reversal strategies available at this point in time.

- The ODls have few drug interactions.

\section{References}

I Keeling D, Baglin T, Tait C et al. British Committee for Standards in Haematology. Guidelines on oral anticoagulation with warfarin, fourth edition. $\mathrm{Br} J$ Haematol 20I I; 154:3 I I-24. http://dx.doi. org/I0.IIIII/j.I365-2 I4I.20II.08753.x

2 Ghanny S, Crowther M. Treatment with novel oral anticoagulants: indications, efficacy and risks. Curr Opin Hematol 20I3; 20:430-6. http://dx.doi.org/10.1097/MOH.0b0I3e328363cl70

3 Baglin T, Keeling D, Kitchen S. Effects on routine coagulation screens and assessment of anticoagulant intensity in patients taking oral dabigatran or rivaroxaban: guidance from the British Committee for Standards in Haematology. Br J Haematol 20I2; I59:427-9. http://dx.doi.org/ I0. I I I I/bjh. I 2052 


\section{SELF-ASSESSMENT QUESTIONS}

\section{Which ONE of the following describes the} mechanism of action of dabigatran?
A. Inhibition of factor VIII.
B. Inhibition of vitamin K-dependent coagulation factors.
C. Activation of fibrinolysis.
D. Inhibition of thrombin.
E. Inhibition of platelet function.

2. Which ONE of the following is the test used to measure warfarin anticoagulant effect?
A. Activated partial thromboplastin time (APTT).
B. Prothrombin time (PT).
C. Thrombin time.
D. Bleeding time.
E. Anti-Xa.

3. Which ONE of the following has a specific reversal strategy?
A. Apixaban.
B. Warfarin.
C. Dabigatran.
D. Rivaroxaban.
E. Fondaparinux.

4. Which ONE of the following drugs may result in suboptimal rivaroxaban concentrations in blood?
A. Ritonavir.
B. Clarithromycin.
C. Aspirin.
D. Phenytoin.
E. Fluconazole.

5. Following the insertion of a prosthetic mitral valve for rheumatic heart disease, which ONE of the following is the anticoagulant of choice?
A. Dabigatran.
B. Rivaroxaban.
C. Warfarin.
D. Apixaban.
E. Clopidogrel.

\section{INVITATION TO SUBMIT PAPERS}

We would like to extend an invitation to all readers of The Journal of the Royal College of Physicians of Edinburgh to contribute original material, especially to the clinical section. The JRCPE is a peer-reviewed journal with a circulation of 8,000 . It is also available open access online. Its aim is to publish a range of clinical, educational and historical material of cross-specialty interest to the College's international membership.

The JRCPE is currently indexed in Medline, Embase, Google Scholar and the Directory of Open Access Journals. The editorial team is keen to continue to improve both the quality of content and its relevance to clinical practice for Fellows and Members. All papers are subject to peer review and our turnaround time for a decision averages only eight weeks.

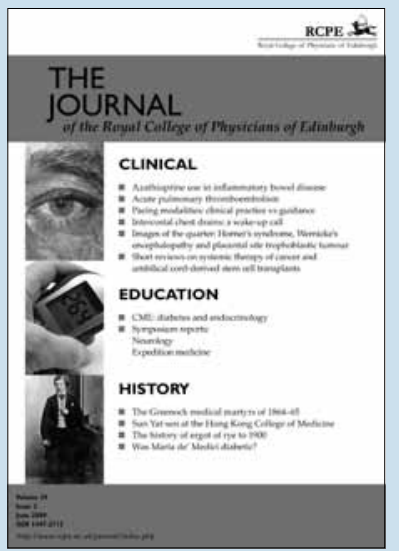

We would be pleased to consider submissions based on original clinical research, including pilot studies. The JRCPE is a particularly good forum for research performed by junior doctors under consultant supervision. We would also consider clinical audits where the 'loop has been closed' and a demonstrable clinical benefit has resulted.

For further information about submissions, please visit: http://www.rcpe.ac.uk/journal/contributers.php or e-mail editorial@rcpe.ac.uk.Thank you for your interest in the College's journal.

The editorial team,

The Journal of the Royal College of Physicians of Edinburgh 